\title{
Fractional Calculus of Analytic Functions Concerned with Möbius Transformations
}

\author{
Nicoleta Breaz, ${ }^{1}$ Daniel Breaz, ${ }^{1}$ and Shigeyoshi Owa ${ }^{2}$ \\ ${ }^{1}$ Department of Mathematics-Informatics, Faculty of Sciences, "1 Decembrie 1918” University of Alba Iulia, 510009 Alba Iulia, Romania \\ ${ }^{2}$ Department of Mathematics, Faculty of Education, Yamato University, Katayama 2-5-1, Suita, Osaka 564-0082, Japan
}

Correspondence should be addressed to Nicoleta Breaz; nicoletabreaz@yahoo.com

Received 17 October 2015; Accepted 3 March 2016

Academic Editor: Simone Secchi

Copyright (C) 2016 Nicoleta Breaz et al. This is an open access article distributed under the Creative Commons Attribution License, which permits unrestricted use, distribution, and reproduction in any medium, provided the original work is properly cited.

Let $\mathscr{A}$ be the class of functions $f(z)$ in the open unit disk $\mathbb{U}$ with $f(0)=0$ and $f^{\prime}(0)=1$. Also, let $w(\zeta)$ be a Möbius transformation in $\mathbb{U}$ for some $z \in \mathbb{U}$. Applying the Möbius transformations, we consider some properties of fractional calculus (fractional derivatives and fractional integrals) of $f(z) \in \mathscr{A}$. Also, some interesting examples for fractional calculus are given.

\section{Introduction}

Let $\mathscr{A}$ denote the class of functions $f(z)$ of the form

$$
f(z)=z+\sum_{k=2}^{\infty} a_{k} z^{k}
$$

which are analytic in the open unit disk $\mathbb{U}=\{z \in \mathbb{C}:|z|<1\}$.

If $f(z) \in \mathscr{A}$ satisfies

$$
\operatorname{Re}\left(\frac{z f^{\prime}(z)}{f(z)}\right)>\alpha \quad(z \in \mathbb{U})
$$

for some real $\alpha(0 \leqq \alpha<1)$, then $f(z)$ is said to be starlike of order $\alpha$ in $\mathbb{U}$. We denote by $\mathcal{S}^{*}(\alpha)$ the class of all starlike functions $f(z)$ of order $\alpha$ in $\mathbb{U}$ and $\mathcal{S}^{*}(0) \equiv \mathcal{S}^{*}$. Furthermore, if $f(z) \in \mathscr{A}$ satisfies

$$
\operatorname{Re}\left(1+\frac{z f^{\prime \prime}(z)}{f^{\prime}(z)}\right)>\alpha \quad(z \in \mathbb{U})
$$

for some real $\alpha(0 \leqq \alpha<0)$, then we say that $f(z)$ is convex of order $\alpha$ in $\mathbb{U}$. We also denote by $\mathscr{K}(\alpha)$ the class of all such functions $f(z)$ and $\mathscr{K}(0) \equiv \mathscr{K}$. In view of definitions for the classes $\mathcal{S}^{*}(\alpha)$ and $\mathscr{K}(\alpha)$, we know that

(i) $f(z) \in \mathscr{K}(\alpha)$ if and only if $z f^{\prime}(z) \in \mathcal{S}^{*}(\alpha)$;

(ii) $f(z) \in \mathcal{S}^{*}(\alpha)$ if and only if $\int_{0}^{z}(f(t) / t) d t \in \mathscr{K}(\alpha)$.
Further, MacGregor [1] and Wilken and Feng [2] have the sharp inclusion relation that $\mathscr{K}(\alpha) \subset \mathcal{S}^{*}(\beta)$ for each $\alpha(0 \leqq$ $\alpha<1$ ) with

$$
\beta= \begin{cases}\frac{1-2 \alpha}{2^{2(1-\alpha)}\left(1-2^{2 \alpha-1}\right)} & \left(\alpha \neq \frac{1}{2}\right) \\ \frac{1}{2 \log 2} & \left(\alpha=\frac{1}{2}\right) .\end{cases}
$$

For $\alpha=0$, Marx [3] and Strohhäcker [4] showed that $\mathscr{K} \subset$ $\mathcal{S}^{*}(1 / 2)$. Also, by Robertson [5], we know that the extremal function $f(z)$ for the class $\mathcal{S}^{*}(\alpha)$ is

$$
f(z)=\frac{z}{(1-z)^{2(1-\alpha)}}=z+\sum_{k=2}^{\infty} \frac{\prod_{j=2}^{k}(j-2 \alpha)}{(k-1) !} z^{k}
$$

and the extremal function $f(z)$ for the class $\mathscr{K}(\alpha)$ is

$$
\begin{aligned}
& f(z) \\
& = \begin{cases}\frac{1-(1-z)^{2 \alpha-1}}{2 \alpha-1}=z+\sum_{k=2}^{\infty} \frac{\prod_{j=2}^{k}(j-2 \alpha)}{k !} z^{k} & \left(\alpha \neq \frac{1}{2}\right) \\
-\log (1-z)=z+\sum_{k=2}^{\infty} \frac{1}{k} z^{k} & \left(\alpha=\frac{1}{2}\right) .\end{cases}
\end{aligned}
$$


For $f(z) \in \mathscr{A}$, we apply the following Möbius transformation:

$$
w(\zeta)=\frac{z+\zeta}{1+\bar{z} \zeta} \quad(\zeta \in \mathbb{U})
$$

for a fixed $z \in \mathbb{U}$. This Möbius transformation $w(\zeta)$ maps $\mathbb{U}$ onto itself and $\zeta=0$ to $w(0)=z$.

\section{Fractional Calculus}

From among the various definitions for fractional calculus (i.e., fractional derivatives and fractional integrals) given in the literature, we have to recall here the following definitions for fractional calculus which are used by Owa [6, 7] and by Owa and Srivastava [8].

Definition 1. The fractional integral of order $\lambda$ is defined, for $f(z) \in \mathscr{A}$, by

$$
D_{z}^{-\lambda} f(z)=\frac{1}{\Gamma(\lambda)} \int_{0}^{z} \frac{f(\zeta)}{(z-\zeta)^{1-\lambda}} d \zeta
$$

where $\lambda>0$ and the multiplicity of $(z-\zeta)^{\lambda-1}$ is removed by requiring $\log (z-\zeta)$ to be real when $z-\zeta>0$.

Definition 2. The fractional derivative of order $\lambda$ is defined, for $f(z) \in \mathscr{A}$, by

$$
\begin{aligned}
D_{z}^{\lambda} f(z) & =\frac{d}{d z}\left(D_{z}^{\lambda-1} f(z)\right) \\
& =\frac{1}{\Gamma(1-\lambda)} \frac{d}{d z} \int_{0}^{z} \frac{f(\zeta)}{(z-\zeta)^{\lambda}} d \zeta
\end{aligned}
$$

where $0 \leqq \lambda<1$ and the multiplicity of $(z-\zeta)^{-\lambda}$ is removed as in Definition 1 above.

Definition 3. Under the hypotheses of Definition 2 , the fractional derivative of order $n+\lambda$ is defined by

$$
D_{z}^{n+\lambda} f(z)=\frac{d^{n}}{d z^{n}}\left(D_{z}^{\lambda} f(z)\right),
$$

where $0 \leqq \lambda<1$ and $n \in \mathbb{N}_{0}=0,1,2, \ldots$

Remark 4. In view of definitions for the fractional calculus of $f(z) \in \mathscr{A}$, we see that

$$
\begin{aligned}
D_{z}^{-\lambda} f(z) & \\
= & \frac{1}{\Gamma(2+\lambda)} z^{1+\lambda}+\frac{2 !}{\Gamma(3+\lambda)} a_{2} z^{2+\lambda}+\cdots \\
& +\frac{k !}{\Gamma(k+1+\lambda)} a_{k} z^{k+\lambda}+\cdots \\
= & \frac{1}{\Gamma(2+\lambda)} z^{1+\lambda}+\sum_{k=2}^{\infty} \frac{k !}{\Gamma(k+1+\lambda)} a_{k} z^{k+\lambda}
\end{aligned}
$$

$(\lambda>0)$

$$
\begin{aligned}
D_{z}^{\lambda} f(z) & \frac{1}{\Gamma(2-\lambda)} z^{1-\lambda}+\frac{2 !}{\Gamma(3-\lambda)} a_{2} z^{2-\lambda}+\cdots \\
& +\frac{k !}{\Gamma(k+1-\lambda)} a_{k} z^{k-\lambda}+\cdots \\
= & \frac{1}{\Gamma(2-\lambda)} z^{1-\lambda}+\sum_{k=2}^{\infty} \frac{k !}{\Gamma(k+1-\lambda)} a_{k} z^{k-\lambda} \\
D_{z}^{n+\lambda} & f(z) \\
= & \frac{d^{n}}{d z^{n}}\left(\frac{1}{\Gamma(2-\lambda)} z^{1-\lambda}+\sum_{k=2}^{\infty} \frac{k !}{\Gamma(k+1-\lambda)} a_{k} z^{k-\lambda}\right) \\
= & \frac{1}{\Gamma(2-n-\lambda)} z^{1-n-\lambda} \quad(0 \leq 1), \\
& +\sum_{k=2}^{\infty} \frac{k !}{\Gamma(k+1-n-\lambda)} a_{k} z^{k-n-\lambda}
\end{aligned}
$$

for $0 \leqq \lambda<1$ and $n \in \mathbb{N}_{0}$.

Therefore, we can write that

$$
\begin{aligned}
D_{z}^{n+\lambda} f(z) & =\frac{d^{n}}{d z^{n}}\left(D_{z}^{\lambda} f(z)\right)=D_{z}^{\lambda}\left(\frac{d^{n}}{d z^{n}} f(z)\right) \\
D_{z}^{\lambda} f(z) & =\frac{1}{\Gamma(2-\lambda)} z^{1-\lambda}+\sum_{k=2}^{\infty} \frac{k !}{\Gamma(k+1-\lambda)} a_{k} z^{k-\lambda}
\end{aligned}
$$

for any real number $\lambda$.

Using the fractional calculus (13), we define

$$
\begin{aligned}
F(z) & =\Gamma(2-\lambda) z^{\lambda} D_{z}^{\lambda} f(z) \\
& =z+\sum_{k=2}^{\infty} \frac{k ! \Gamma(2-\lambda)}{\Gamma(k+1-\lambda)} a_{k} z^{k} \quad(\lambda \in \mathbb{R}, \lambda \neq 2) .
\end{aligned}
$$

If we take $\lambda=-1$ in (14), then

$$
\begin{aligned}
F(z) & =\Gamma(3) z^{-1} D_{z}^{-1} f(z)=\frac{2}{z} \int_{0}^{z} f(t) d t \\
& =z+\sum_{k=2}^{\infty} \frac{2}{k+1} a_{k} \cdot z^{k}
\end{aligned}
$$

implies the Libera integral operator defined by Libera [9]. Therefore, $F(z)$ given by (14) is the generalization operator of Libera integral operator.

Let us give two examples for the fractional operator $F(z)$ defined in (14).

Example 5. Let us define $f(z)$ by

$$
f(z)=z+\frac{2-\lambda}{6} z^{2} \in \mathscr{A} \quad(-1 \leqq \lambda<2) .
$$


Then, we have that

$$
\begin{aligned}
\operatorname{Re}\left(\frac{z f^{\prime}(z)}{f(z)}\right) & =\operatorname{Re}\left(2-\frac{1}{1+M z}\right) \\
& =2-\frac{1+M \cos \theta}{1+M^{2}+2 M \cos \theta} \quad\left(z=e^{i \theta}\right),
\end{aligned}
$$

where $M=(2-\lambda) / 6>0$. If we define

$$
h(t)=\frac{1+M t}{1+M^{2}+2 M t} \quad(t=\cos \theta),
$$

then

$$
h^{\prime}(t)=\frac{M(M+1)(M-1)}{\left(1+M^{2}+2 M t\right)^{2}}<0 \quad\left(0<M \leqq \frac{1}{2}\right) .
$$

This shows us that

$$
h(t) \leqq h(-1)=\frac{1}{1-M} .
$$

That is,

$$
\operatorname{Re}\left(\frac{z f^{\prime}(z)}{f(z)}\right)>2-\frac{1}{1-M}=\frac{2+2 \lambda}{4+\lambda}>0 \quad(z \in \mathbb{U}) .
$$

Therefore, $f(z) \in \mathcal{S}^{*}((2+2 \lambda) /(4+\lambda))$.

For $f(z)$, given by (16), $F(z)$ becomes

$$
F(z)=\Gamma(2-\lambda) z^{\lambda} D_{z}^{\lambda} f(z)=z+\frac{1}{3} z^{2}
$$

$$
(-1 \leqq \lambda<2) .
$$

Then, we see that $F(z) \in \mathcal{S}^{*}(1 / 2)$.

Next, let us consider the function $g(\zeta)$ given by

$$
g(\zeta)=\frac{(F \circ w)(\zeta)-F(z)}{\left(1-|z|^{2}\right) F^{\prime}(z)} \quad(\zeta \in \mathbb{U})
$$

for a fixed $z \in \mathbb{U}$, where $w(\zeta)$ is given by (7). Then, it is easy to see that $g(\zeta) \in \mathscr{A}$. Taking $z=1 / 2$ in (23), we have that

$$
\begin{aligned}
& g(\zeta)=\frac{\zeta(11 \zeta+16)}{4(\zeta+2)^{2}} \quad(\zeta \in \mathbb{U}), \\
& \operatorname{Re}\left(\frac{\zeta g^{\prime}(\zeta)}{g(\zeta)}\right)=\operatorname{Re}\left(1-\frac{\zeta(11 \zeta+10)}{(\zeta+2)(11 \zeta+16)}\right) \\
&=1-\frac{704 \cos ^{2} \theta+848 \cos \theta+149}{1408 \cos ^{2} \theta+3268 \cos \theta+1885} \\
&\left(\theta=e^{i \theta}\right) .
\end{aligned}
$$

Letting

$$
H(t)=\frac{704 t^{2}+848 t+149}{1408 t^{2}+3268 t+1885} \quad(t=\cos \theta),
$$

we obtain that

$$
H^{\prime}(t)=\frac{12\left(9224 t^{2}+186208 t+92629\right)}{\left(1408 t^{2}+3268 t+1885\right)^{2}} .
$$

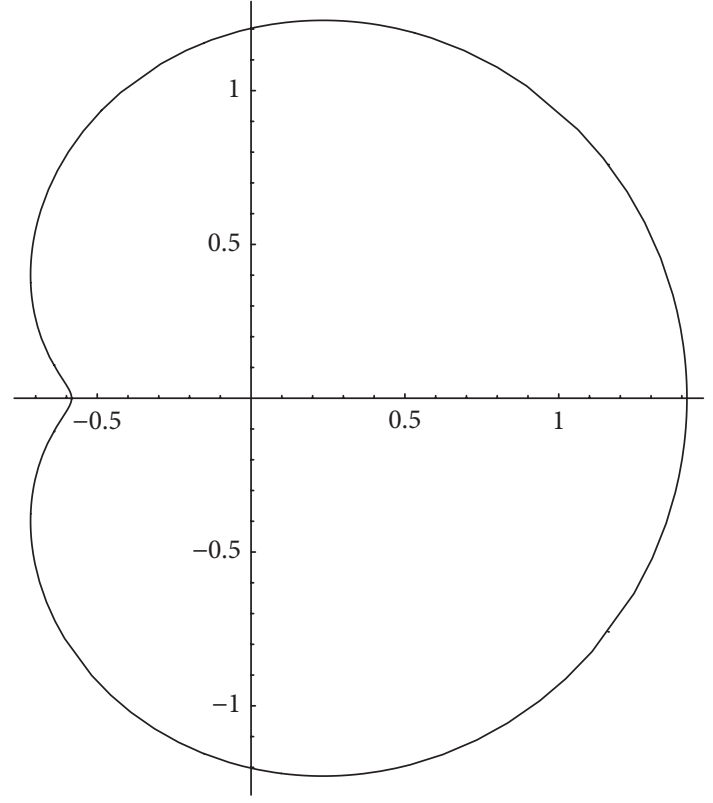

FIGURE 1

This shows that $H^{\prime}(-1)<0, H^{\prime}(0)>0$, and $H^{\prime}(1)>0$. Therefore, there exists some $t_{0}$ such that $H^{\prime}\left(t_{0}\right)=0$ for $-1<t_{0}<0$. It follows that

$$
\max _{-1 \leqq t \leqq 1} H(t)=\max \{H(-1), H(1)\}=H(1)=\frac{7}{27} .
$$

Thus, we say that

$$
\operatorname{Re}\left(\frac{\zeta g^{\prime}(\zeta)}{g(\zeta)}\right)>1-\frac{7}{27}=\frac{20}{27} \quad(\zeta \in \mathbb{U})
$$

Consequently, we say that $F(z) \in \mathcal{S}^{*}(1 / 2), g(\zeta) \in \mathcal{S}^{*}(20 / 27)$ for $f(z) \in \mathcal{S}^{*}((2+2 \lambda) /(4+\lambda))$ given by $(16)$.

If $\lambda=-1 / 2$, then

$$
f(z)=z+\frac{5}{12} z^{2} \in \mathcal{S}^{*}\left(\frac{2}{7}\right) .
$$

The open unit disk $\mathbb{U}$ is mapped on the starlike domain of order $2 / 7$ in Figure 1.

If $\lambda=1 / 3$, then

$$
f(z)=z+\frac{5}{18} z^{2} \in \mathcal{S}^{*}\left(\frac{8}{13}\right) .
$$

Thus, $f(z)$ maps $\mathbb{U}$ onto the starlike domain of order $8 / 13$ in Figure 2 .

Example 5 means that there is some function $f(z) \in$ $\mathcal{S}^{*}(\alpha)$ such that $F(z) \in \mathcal{S}^{*}(\beta)$ and $g(\zeta) \in \mathcal{S}^{*}(\gamma)$.

Next, we consider the following.

Example 6. Let a function $f(z)$ be given by

$$
f(z)=z+\frac{2-\lambda}{12} z^{2} \in \mathscr{A} \quad(-1 \leqq \lambda<2) .
$$




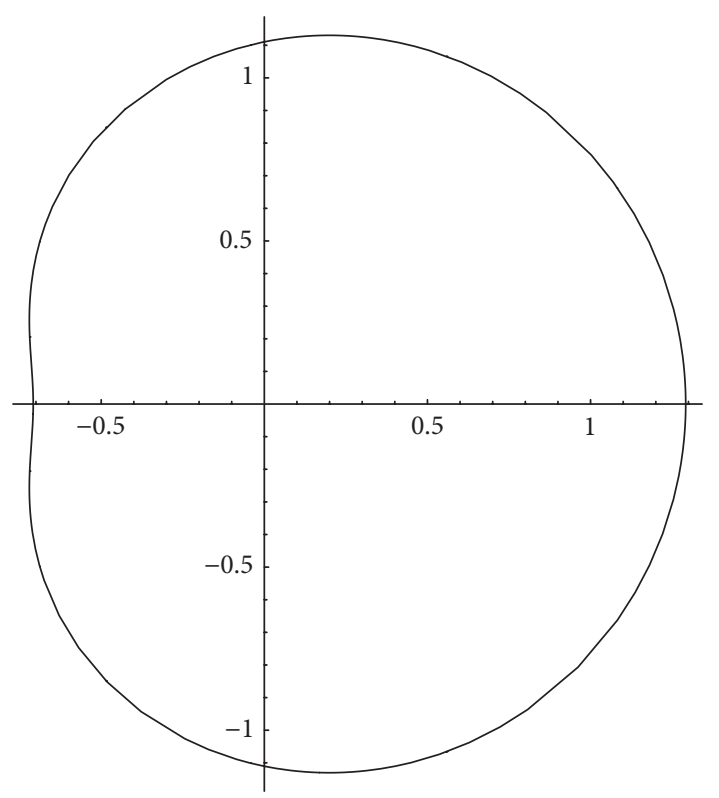

FIgURE 2

Then, we have that

$$
\begin{aligned}
\operatorname{Re}\left(1+\frac{z f^{\prime \prime}(z)}{f^{\prime}(z)}\right) & =\operatorname{Re}\left(2-\frac{1}{1+2 M z}\right) \\
& =2-\frac{1+2 M \cos \theta}{1+4 M^{2}+4 M \cos \theta} \\
& \left(z=e^{i \theta}\right),
\end{aligned}
$$

where $M=(2-\lambda) / 12>0$. Defining $h(t)$ by

$$
h(t)=\frac{1+2 M t}{1+4 M^{2}+4 M t} \quad(t=\cos \theta)
$$

we have that

$$
h^{\prime}(t)=\frac{2 M(2 M+1)(2 M-1)}{\left(1+4 M^{2}+4 M t\right)^{2}}<0 \quad\left(0<M \leqq \frac{1}{4}\right)
$$

which shows us that

$$
h(t) \leqq h(-1)=\frac{1}{1-2 M} .
$$

Thus, we obtain that

$$
\operatorname{Re}\left(1+\frac{z f^{\prime \prime}(z)}{f^{\prime}(z)}\right)>2-\frac{1}{1-2 M}=\frac{2+2 \lambda}{4+\lambda}>0
$$

$(z \in \mathbb{U})$.

This gives us that $f(z) \in \mathscr{K}((2+2 \lambda) /(4+\lambda))$.

For $f(z)$, given by (31), $F(z)$ becomes

$$
\begin{aligned}
& F(z)=\Gamma(2-\lambda) z^{\lambda} D_{z}^{\lambda} f(z)=z+\frac{1}{6} z^{2} \\
& \quad(-1 \leqq \lambda<2) .
\end{aligned}
$$

Then, it is easy to see that $F(z) \in \mathscr{K}(1 / 2)$.
For this $F(z)$, we consider $g(\zeta)$ defined by (23). If we take $z=1 / 2$ for $g(\zeta)$, we have that

$$
\begin{aligned}
& g(\zeta)=\frac{\zeta(17 \zeta+28)}{7(\zeta+2)^{2}} \quad(\zeta \in \mathbb{U}), \\
& \operatorname{Re}\left(1+\frac{\zeta g^{\prime \prime}(\zeta)}{g^{\prime}(\zeta)}\right)=\operatorname{Re}\left(1-\frac{\zeta(10 \zeta+11)}{(5 \zeta+7)(\zeta+2)}\right) \\
&=1-\frac{280 \cos ^{2} \theta+379 \cos \theta+97}{280 \cos ^{2} \theta+646 \cos \theta+370} \\
&\left(\zeta=e^{i \theta}\right) .
\end{aligned}
$$

If we write that

$$
H(t)=\frac{280 t^{2}+379 t+97}{280 t^{2}+646 t+370} \quad(t=\cos \theta),
$$

then

$$
H^{\prime}(t)=\frac{24\left(3115 t^{2}+6370 t+3232\right)}{\left(280 t^{2}+646 t+370\right)^{2}} .
$$

Since $H^{\prime}(-1)<0, H^{\prime}(0)>0$, and $H^{\prime}(1)>0$, there exists some $t_{0}$ such that $H^{\prime}\left(t_{0}\right)=0$ for $-1<t_{0}<0$. This gives us that

$$
\max _{-1 \leqq t \leqq 1} H(t)=\max \{H(-1), H(1)\}=H(1)=\frac{7}{12} .
$$

It follows that

$$
\operatorname{Re}\left(1+\frac{\zeta g^{\prime \prime}(\zeta)}{g^{\prime}(\zeta)}\right)>1-\frac{7}{12}=\frac{5}{12} \quad(\zeta \in \mathbb{U}) .
$$

Therefore, we say that $F(z) \in \mathscr{K}(1 / 2), g(\zeta) \in \mathscr{K}(5 / 12)$ for $f(z) \in \mathscr{K}((2+2 \lambda) /(4+\lambda))$.

If $\lambda=-2 / 3$, then

$$
f(z)=z+\frac{2}{9} z^{2} \in \mathscr{K}\left(\frac{1}{5}\right)
$$

maps $\mathbb{U}$ onto the convex domain of order $1 / 5$ in Figure 3.

If $\lambda=3 / 2$, then

$$
f(z)=z+\frac{1}{24} z^{2} \in \mathscr{K}\left(\frac{10}{11}\right) .
$$

This function $f(z)$ maps $\mathbb{U}$ onto the convex domain of order 10/11 in Figure 4.

Example 6 says that there exists some function $f(z) \in$ $\mathscr{K}(\alpha)$ such that $F(z) \in \mathscr{K}(\beta)$ and $g(\zeta) \in \mathscr{K}(\gamma)$.

In view of Examples 5 and 6, we introduce the following.

Definition 7. Let $f(z) \in \mathscr{A}, F(z)=\Gamma(2-\lambda) z^{\lambda} D_{z}^{\lambda} f(z)$ with $-1 \leqq \lambda<2$ and let $g(\zeta)$ be defined by (23) for a fixed $z \in \mathbb{U}$. Then, we say that

(i) $f(z) \in \mathcal{S}_{0}$ if $g(\zeta)$ is univalent in $\mathbb{U}$,

(ii) $f(z) \in \mathcal{S}_{0}^{*}(\alpha)$ if $g(\zeta) \in \mathcal{S}^{*}(\alpha)$,

(iii) $f(z) \in \mathscr{K}_{0}(\alpha)$ if $g(\zeta) \in \mathscr{K}(\alpha)$. 


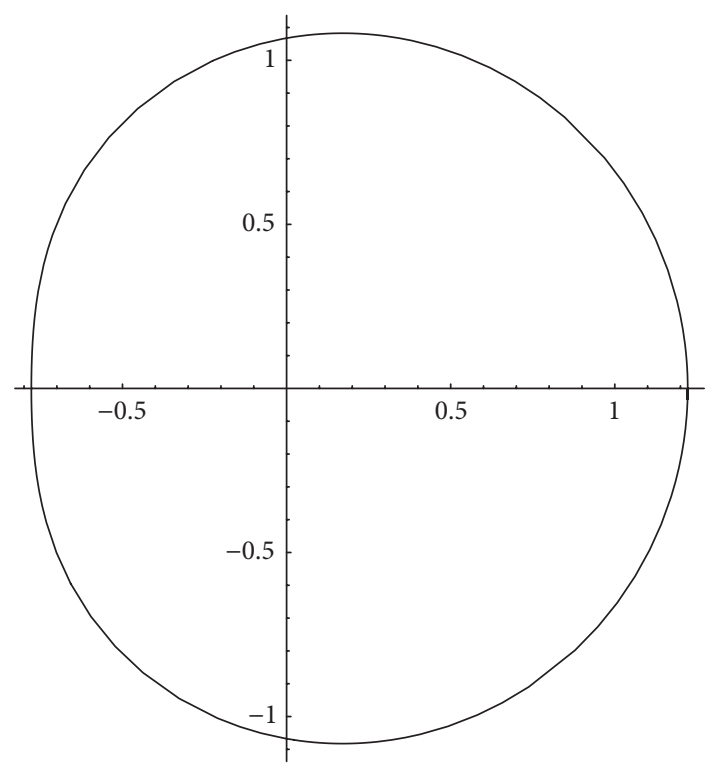

FIgURE 3

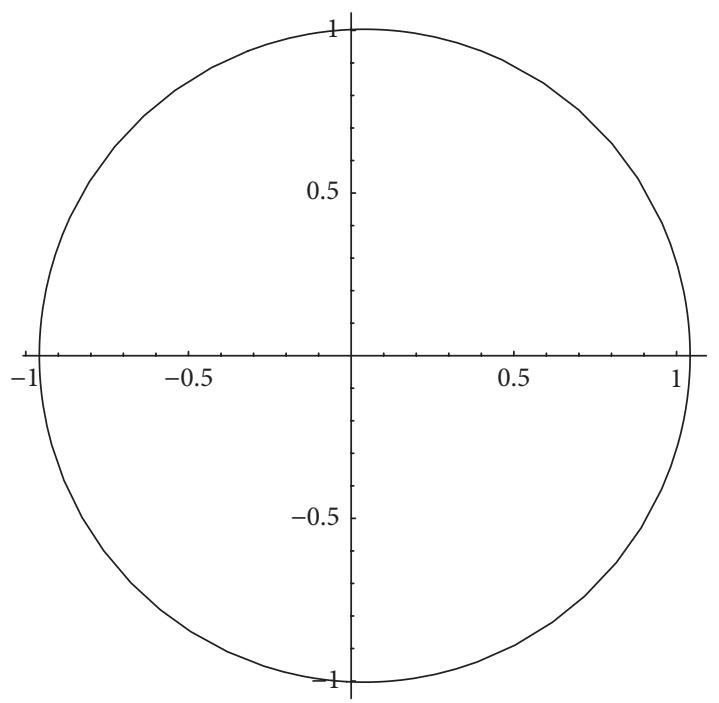

FigURE 4
Also, we write that $\mathcal{S}_{0}^{*}(0) \equiv \mathcal{S}_{0}^{*}$ and $\mathscr{K}_{0}(0) \equiv \mathscr{K}_{0}$ when $\alpha=0$.

In order to discuss our classes $\mathcal{S}_{0}, \mathcal{S}_{0}^{*}(\alpha)$, and $\mathscr{K}_{0}(\alpha)$, we need the following lemma due to Robertson [5] (also see Duren [10]).

Lemma 8. If $f(z) \in \mathcal{S}^{*}(\alpha)$, then

$$
\left|a_{k}\right| \leqq \frac{\prod_{j=2}^{k}(j-2 \alpha)}{(k-1) !} \quad(k=2,3,4, \ldots)
$$

with the equality in (45) with $f(z)$ given by (5). If $f(z) \in \mathscr{K}(\alpha)$, then

$$
\left|a_{k}\right| \leqq \frac{\prod_{j=2}^{k}(j-2 \alpha)}{k !} \quad(k=2,3,4, \ldots)
$$

with the equality in (46) with $f(z)$ given by (6).

We also need the following lemma.

Lemma 9. If $g(\zeta)$ is defined by

$$
g(\zeta)=\frac{(f \circ w)(\zeta)-f(z)}{\left(1-|z|^{2}\right) f^{\prime}(z)} \quad(\zeta \in \mathbb{U})
$$

for a fixed $z \in \mathbb{U}$ for $f(z) \in \mathscr{A}$, then

$$
\begin{aligned}
& \frac{\left(d^{n} / d \zeta^{n}\right)(f \circ w)(\zeta)}{f^{\prime}(z)} \\
& =\frac{n !(n-1) !(1+\bar{z} \zeta)^{2 n}}{\left(1-|z|^{2}\right)^{n-1}}\left(\sum_{j=0}^{n-1} \frac{g^{(n-j)}(\zeta) \bar{z}^{j}}{(n-j) !(n-j-1) ! j !(1+\bar{z} \zeta)^{j}}\right)
\end{aligned}
$$

for $n=1,2,3, \ldots$, where $w(\zeta)$ is given by $(7)$.

Proof. We use the mathematical induction to prove (48). For $n=1$, the right-hand side of (48) becomes

$$
(1+\bar{z} \zeta)^{2} g^{\prime}(\zeta)=\frac{(d / d \zeta)(f \circ w)(\zeta)}{f^{\prime}(z)}
$$

Therefore, (48) holds true for $n=1$.

Assume that relation (48) is true for a fixed positive integer $n$. Then, some calculations lead us to

$$
\begin{aligned}
& \frac{\left(d^{n+1} / d \zeta^{n+1}\right)(f \circ w)(\zeta)\left(1-|z|^{2}\right)}{(1+\bar{z} \zeta)^{2} f^{\prime}(z)} \\
& \quad=\frac{n !(n-1) !(1+\bar{z} \zeta)^{2 n}}{\left(1-|z|^{2}\right)^{n-1}}\left\{\sum_{j=0}^{n-1} \frac{1}{(n-j) !(n-j-1) ! j !}\left(g^{(n+1-j)}(\zeta)+\frac{(2 n-j) g^{(n-j)}(\zeta) \bar{z}}{1+\bar{z} \zeta}\right) \frac{\bar{z}^{j}}{(1+\bar{z} \zeta)^{j}}\right\} \\
& \quad=\frac{(n+1) ! n !(1+\bar{z} \zeta)^{2 n}}{\left(1-|z|^{2}\right)^{n-1}}\left(\sum_{j=0}^{n} \frac{g^{(n+1-j)}(\zeta) \bar{z}^{j}}{(n+1-j) !(n-j) ! j !(1+\bar{z} \zeta)^{j}}\right) .
\end{aligned}
$$


This means that relation (48) holds true for $n+1$. Thus, by applying the mathematical induction, we complete the proof of the lemma.

Taking $\zeta=0$ in Lemma 9, we have the following.

Corollary 10. If $g(\zeta)$ is defined by (47) for $f(z) \in \mathscr{A}$, then one has

$$
\begin{aligned}
& \left|\frac{f^{(n)}(z)}{f^{\prime}(z)}\right| \\
& \quad \leqq \frac{n !(n-1) !}{\left(1-|z|^{2}\right)^{n-1}}\left(\sum_{j=0}^{n-1} \frac{\left|g^{(n-j)}(0)\right||z|^{j}}{(n-j) !(n-j-1) ! j !}\right)
\end{aligned}
$$

for $z \in \mathbb{U}$. Furthermore, we have

$$
\left|\frac{f^{\prime \prime}(z)}{f^{\prime}(z)}\right| \leqq \frac{\left|g^{\prime \prime}(0)\right|+2\left|g^{\prime}(0)\right||z|}{1-|z|^{2}} \quad(z \in \mathbb{U}) .
$$

Applying Corollary 10, we have the following.

Theorem 11. Let $F(z)$ be defined by (14) for $f(z) \in \mathscr{A}$ with $-1 \leqq \lambda<2$.

(i) If $f(z) \in \mathcal{S}_{0}$, then

$$
\left|\frac{F^{(n)}(z)}{F^{\prime}(z)}\right| \leqq \frac{n !(n+|z|)}{(1-|z|)^{n-1}(1+|z|)} \quad(n=1,2,3, \ldots)
$$

with the equality for $g(\zeta)$ given by

$$
g(\zeta)=\frac{\zeta}{\left(1+e^{i \theta} \zeta\right)^{2}} \quad(\theta \in \mathbb{R}) .
$$

(ii) If $f(z) \in \mathcal{S}_{0}^{*}(\alpha)$, then

$$
\begin{aligned}
& \left|\frac{F^{(n)}(z)}{F^{\prime}(z)}\right| \\
& \quad \leqq \frac{n !(n-1) !}{\left(1-|z|^{2}\right)^{n-1}}\left(\sum_{j=0}^{n-1} \frac{\prod_{k=2}^{n-j}(k-2 \alpha)}{j !((n-j-1) !)^{2}}|z|^{j}\right)
\end{aligned}
$$

with the equality for $g(\zeta)$ given by

$$
g(\zeta)=\frac{\zeta}{\left(1+e^{i \theta} \zeta\right)^{2(1-\alpha)}} \quad(\theta \in \mathbb{R})
$$

(iii) If $f(z) \in \mathscr{K}_{0}(\alpha)$, then

$$
\begin{aligned}
& \left|\frac{F^{(n)}(z)}{F^{\prime}(z)}\right| \\
& \leqq \frac{n !(n-1) !}{\left(1-|z|^{2}\right)^{n-1}}\left(\sum_{j=0}^{n-1} \frac{\prod_{k=2}^{n-j}(k-2 \alpha)}{j !(n-j) !(n-j-1) !}|z|^{j}\right) \\
& \quad(n=1,2,3, \ldots)
\end{aligned}
$$

with the equality for $g(\zeta)$ given by

$$
g(\zeta)= \begin{cases}\frac{1-(1-\zeta)^{2 \alpha-1}}{2 \alpha-1} & \left(\alpha \neq \frac{1}{2}\right) \\ -\log (1-\zeta) & \left(\alpha=\frac{1}{2}\right)\end{cases}
$$

Proof. Note that

$$
g(\zeta)=\frac{(F \circ w)(\zeta)-F(z)}{\left(1-|z|^{2}\right) F^{\prime}(z)} \quad(\zeta \in \mathbb{U})
$$

for $F(z)=\Gamma(2-\lambda) z^{\lambda} D_{z}^{\lambda} f(z)$. Therefore, Corollary 10 gives us that

$$
\begin{aligned}
& \left|\frac{F^{(n)}(z)}{F^{\prime}(z)}\right| \\
& \quad \leqq \frac{n !(n-1) !}{\left(1-|z|^{2}\right)^{n-1}}\left(\sum_{j=0}^{n-1} \frac{\left|g^{(n-j)}(0)\right||z|^{j}}{(n-j) !(n-j-1) ! j !}\right) .
\end{aligned}
$$

According to Lemma 8, we have

$$
\left|g^{(n-j)}(0)\right| \leqq(n-j) !(n-j)
$$

if $f(z) \in \mathcal{S}_{0}$, then we calculate that

$$
\begin{aligned}
\left|\frac{F^{(n)}(z)}{F^{\prime}(z)}\right| & \leqq \frac{n !(n-1) !}{\left(1-|z|^{2}\right)^{n-1}}\left(\sum_{j=0}^{n-1} \frac{n-j}{(n-j-1) ! j !}|z|^{j}\right) \\
& =\frac{n !(n+|z|)}{(1-|z|)^{n-1}(1+|z|)},
\end{aligned}
$$

because

$$
\sum_{j=0}^{n-1} \frac{n-j}{(n-j-1) ! j !}|z|^{j}=\frac{(n+|z|)(1+|z|)^{n-2}}{(n-1) !} .
$$

If $f(z) \in \mathcal{S}_{0}^{*}(\alpha)$, then

$$
\left|g^{(n-j)}(0)\right| \leqq(n-j) \prod_{k=2}^{n-j}(k-2 \alpha) \quad\left(g^{\prime}(0)=1\right)
$$

by means of (45). This implies inequality (55) for $f(z) \in$ $\delta_{0}^{*}(\alpha)$. Furthermore, if $f(z) \in \mathscr{K}_{0}(\alpha)$, then $g(\zeta)$ satisfies

$$
\left|g^{(n-j)}(0)\right| \leqq \prod_{k=2}^{n-j}(k-2 \alpha) \quad\left(g^{\prime}(0)=1\right)
$$

which implies inequality (57). Consequently, we complete the proof of the theorem. 
Since $g^{\prime}(0)=1$, letting $n=2$ in Theorem 11, we have the following.

Corollary 12. Let $f(z) \in \mathscr{A}$ with $-1 \leqq \lambda<2$.

(i) If $f(z) \in \mathcal{S}_{0}$, then

$$
\begin{aligned}
& \left|\frac{\lambda(\lambda-1) D_{z}^{\lambda} f(z)+2 \lambda z D_{z}^{\lambda+1} f(z)+z^{2} D_{z}^{\lambda} f(z)}{z\left(\lambda D_{z}^{\lambda} f(z)+z D_{z}^{\lambda+1} f(z)\right)}\right| \\
& \quad \leqq \frac{2(2+|z|)}{1-|z|^{2}} \\
& \quad \text { for } z \in \mathbb{U} .
\end{aligned}
$$

(ii) If $f(z) \in \mathcal{S}_{0}^{*}(\alpha)$, then

$$
\begin{aligned}
& \left|\frac{\lambda(\lambda-1) D_{z}^{\lambda} f(z)+2 \lambda z D_{z}^{\lambda+1} f(z)+z^{2} D_{z}^{\lambda} f(z)}{z\left(\lambda D_{z}^{\lambda} f(z)+z D_{z}^{\lambda+1} f(z)\right)}\right| \\
& \quad \leqq \frac{2(2(1-\alpha)+|z|)}{1-|z|^{2}}
\end{aligned}
$$

for $z \in \mathbb{U}$.

(iii) If $f(z) \in \mathscr{K}_{0}(\alpha)$, then

$$
\begin{aligned}
& \left|\frac{\lambda(\lambda-1) D_{z}^{\lambda} f(z)+2 \lambda z D_{z}^{\lambda+1} f(z)+z^{2} D_{z}^{\lambda} f(z)}{z\left(\lambda D_{z}^{\lambda} f(z)+z D_{z}^{\lambda+1} f(z)\right)}\right| \\
& \quad \leqq \frac{2(1-\alpha+|z|)}{1-|z|^{2}} \\
& \quad \text { for } z \in \mathbb{U} .
\end{aligned}
$$

Taking $\lambda=0$ in Corollary 12, we have the following.

Corollary 13. If $f(z) \in \mathcal{S}_{0}$, then

$$
\left|\frac{f(z)}{f^{\prime}(z)}\right| \leqq \frac{2(2+|z|)}{1-|z|^{2}} \quad(z \in \mathbb{U}),
$$

if $f(z) \in \mathcal{S}_{0}^{*}(\alpha)$, then

$$
\left|\frac{f(z)}{f^{\prime}(z)}\right| \leqq \frac{2(2(1-\alpha)+|z|)}{1-|z|^{2}} \quad(z \in \mathbb{U}),
$$

and if $f(z) \in \mathscr{K}_{0}(\alpha)$, then

$$
\left|\frac{f(z)}{f^{\prime}(z)}\right| \leqq \frac{2(1-\alpha+|z|)}{1-|z|^{2}} \quad(z \in \mathbb{U}) .
$$

\section{Univalence of Fractional Calculus}

Let $f(z)$ and $g(z)$ be analytic in $\mathbb{U}$. Then, $f(z)$ is said to be subordinate to $g(z)$, written $f(z) \prec g(z)$, if there exists a function $w(z)$ analytic in $\mathbb{U}$ with $w(0)=0$ and $|w(z)|<1(z \in$ $\mathbb{U})$, such that $f(z)=g(w(z))$. Furthermore, if $g(z)$ is univalent in $\mathbb{U}$, then the subordination $f(z) \prec g(z)$ is equivalent to $f(0)=g(0)$ and $f(\mathbb{U}) \subset g(\mathbb{U})$ (cf. Miller and Mocanu [11]).

To discuss the univalence of fractional calculus $F(z)$ given by (14), we need the following lemma due to Miller and Mocanu [12] (or due to Jack [13]).
Lemma 14. Let the function $w(z)$ be analytic in $\mathbb{U}$ with $w(0)=$ 0 . If there exists a point $z_{0} \in \mathbb{U}$ such that

$$
\max _{|z| \leqq z_{0} \mid}|w(z)|=\left|w\left(z_{0}\right)\right|,
$$

then

$$
\frac{z_{0} w^{\prime}\left(z_{0}\right)}{w\left(z_{0}\right)}=k
$$

$$
\operatorname{Re}\left(1+\frac{z_{0} w^{\prime \prime}\left(z_{0}\right)}{w^{\prime}\left(z_{0}\right)}\right) \geqq k
$$

where $k \geqq 1$.

Now, we derive the following.

Theorem 15. If $F(z)$ defined by (14) for $f(z) \in \mathscr{A}$ satisfies

$$
\left|1+\frac{1}{2} \frac{z F^{\prime \prime}(z)}{F^{\prime}(z)}-\frac{z F^{\prime}(z)}{F(z)}\right|<\frac{2-\alpha}{4 \alpha} \quad(z \in \mathbb{U})
$$

for some real $\alpha$ which satisfies $2(\sqrt{2}-1) \leqq \alpha<1$, then

$$
\frac{z^{2} F^{\prime}(z)}{F(z)^{2}} \prec \frac{1+(1-\alpha) z}{1-z} \quad(z \in \mathbb{U}) .
$$

Proof. We define the function $w(z)$ by

$$
\frac{z^{2} F^{\prime}(z)}{F(z)^{2}}=\frac{1+(1-\alpha) w(z)}{1-w(z)} \quad(z \in \mathbb{U})
$$

with $2(\sqrt{2}-1) \leqq \alpha<1$. Then, we see that $w(z)$ is analytic in $\mathbb{U}$ with $w(0)=0$ and that

$$
\begin{aligned}
1 & +\frac{1}{2} \frac{z F^{\prime \prime}(z)}{F^{\prime}(z)}-\frac{z F^{\prime}(z)}{F(z)} \\
& =\frac{z w^{\prime}(z)}{2}\left(\frac{1-\alpha}{1+(1-\alpha) w(z)}+\frac{1}{1-w(z)}\right) .
\end{aligned}
$$

If there exists a point $z_{0} \in \mathbb{U}$ such that

$$
\max _{|z| \leqq z_{0} \mid}|w(z)|=\left|w\left(z_{0}\right)\right|=1,
$$

then Lemma 14 gives us that

$$
z_{0} w^{\prime}\left(z_{0}\right)=k w\left(z_{0}\right) \quad(k \geqq 1)
$$

and $w\left(z_{0}\right)=e^{i \theta}$. This implies that

$$
\begin{aligned}
\mid 1 & +\frac{1}{2} \frac{z_{0} F^{\prime \prime}\left(z_{0}\right)}{F^{\prime}\left(z_{0}\right)}-\frac{z_{0} F^{\prime}\left(z_{0}\right)}{F\left(z_{0}\right)} \mid \\
& =\frac{k}{2}\left|\frac{1-\alpha}{1+(1-\alpha) e^{i \theta}}+\frac{1}{1-e^{i \theta}}\right| \\
& \geqq \frac{2-\alpha}{2}\left|\frac{1}{\left(1-e^{i \theta}\right)\left(1+(1-\alpha) e^{i \theta}\right)}\right|=\frac{2-\alpha}{2} \\
& \cdot \frac{1}{\sqrt{2(1-\cos \theta)\left(1+(1-\alpha)^{2}+2(1-\alpha) \cos \theta\right)}} .
\end{aligned}
$$


Letting

$$
g(t)=(1-t)\left(1+(1-\alpha)^{2}+2(1-\alpha) t\right)
$$

we have that

$$
g^{\prime}(t)=-\left(\alpha^{2}+4(1-\alpha) t\right) \leqq-\left(\alpha^{2}+4 \alpha-4\right) \leqq 0
$$

for $2(\sqrt{2}-1) \leqq \alpha<1$. Thus,

$$
g(t) \leqq g(-1)=2 \alpha^{2} .
$$

Therefore, $F(z)$ satisfies

$$
\left|1+\frac{1}{2} \frac{z_{0} F^{\prime \prime}\left(z_{0}\right)}{F^{\prime}\left(z_{0}\right)}-\frac{z_{0} F^{\prime}\left(z_{0}\right)}{F\left(z_{0}\right)}\right| \geqq \frac{2-\alpha}{4 \alpha}
$$

for $2(\sqrt{2}-1) \leqq \alpha<1$. This contradicts our condition (74) for $F(z)$. Thus, $w(z)$ satisfies $|w(z)|<1$ for all $z \in \mathbb{U}$. With this reason above, we conclude that

$$
\frac{z^{2} F^{\prime}(z)}{F(z)^{2}} \prec \frac{1+(1-\alpha) z}{1-z} \quad(z \in \mathbb{U}) .
$$

Next, we show the following.

Theorem 16. If $F(z)$ defined by (14) for $f(z) \in \mathscr{A}$ satisfies

$$
\left|1+\frac{1}{2} \frac{z F^{\prime \prime}(z)}{F^{\prime}(z)}-\frac{z F^{\prime}(z)}{F(z)}\right|<\frac{\alpha}{2(1+\alpha)} \quad(z \in \mathbb{U})
$$

for some real $\alpha>0$, then

$$
\left|\frac{z^{2} F^{\prime}(z)}{F(z)^{2}}-1\right|<\alpha \quad(z \in \mathbb{U}) .
$$

Proof. Let us define the function $w(z)$ by

$$
\frac{z^{2} F^{\prime}(z)}{F(z)^{2}}-1=\alpha w(z) \quad(z \in \mathbb{U}) .
$$

Then,

$$
\begin{aligned}
\mid 1 & +\frac{1}{2} \frac{z F^{\prime \prime}(z)}{F^{\prime}(z)}-\frac{z F^{\prime}(z)}{F(z)}\left|=\frac{\alpha}{2}\right| \frac{z w^{\prime}(z)}{1+\alpha w(z)} \mid \\
& <\frac{\alpha}{2(1+\alpha)} \quad(z \in \mathbb{U}) .
\end{aligned}
$$

Suppose that there exists a point $z_{0} \in \mathbb{U}$ such that

$$
\max _{|z| \leq\left|z_{0}\right|}|w(z)|=\left|w\left(z_{0}\right)\right|=1,
$$

then we can write that

$$
z_{0} w^{\prime}\left(z_{0}\right)=k w\left(z_{0}\right) \quad(k \geqq 1)
$$

and $w\left(z_{0}\right)=e^{i \theta}$. Therefore, we have that

$$
\begin{aligned}
\mid 1 & +\frac{1}{2} \frac{z_{0} F^{\prime \prime}\left(z_{0}\right)}{F^{\prime}\left(z_{0}\right)}-\frac{z_{0} F^{\prime}\left(z_{0}\right)}{F\left(z_{0}\right)}\left|=\frac{k \alpha}{2}\right| \frac{1}{1+\alpha e^{i \theta}} \mid \\
& \geqq \frac{\alpha}{2(1+\alpha)},
\end{aligned}
$$

which contradicts our condition (86). Thus, we say that there is no $z_{0} \in \mathbb{U}$ such that $\left|w\left(z_{0}\right)\right|=1$. Consequently, letting $|w(z)|<1$ for all $z \in \mathbb{U}$, we prove the theorem.

Taking $\alpha=1$ in Theorem 16, we have

Corollary 17. If $F(z)$ defined by (14) for $f(z) \in \mathscr{A}$ satisfies

$$
\left|1+\frac{1}{2} \frac{z F^{\prime \prime}(z)}{F^{\prime}(z)}-\frac{z F^{\prime}(z)}{F(z)}\right|<\frac{1}{4} \quad(z \in \mathbb{U}),
$$

then

$$
\left|\frac{z^{2} F^{\prime}(z)}{F(z)^{2}}-1\right|<1 \quad(z \in \mathbb{U}) .
$$

Remark 18. In view of the result for the univalence of analytic functions due to Ozaki and Nunokawa [14], we see that $F(z)$ satisfying inequality (94) is univalent in $\mathbb{U}$.

Example 19. Let us consider the function $f(z)$ given by

$$
f(z)=D_{z}^{-\lambda}\left(\frac{z^{1-\lambda}}{\Gamma(2-\lambda)} e^{z / 2}\right) \quad(-1 \leqq \lambda<2) .
$$

Then, we have that

$$
\begin{aligned}
F(z) & =\Gamma(2-\lambda) z^{\lambda} D_{z}^{\lambda} f(z)=z e^{z / 2}, \\
\frac{z F^{\prime}(z)}{F(z)} & =1+\frac{1}{2} z, \\
\frac{z F^{\prime \prime}(z)}{F^{\prime}(z)} & =\frac{1}{2} z+\frac{z}{2+z} .
\end{aligned}
$$

Therefore, $F(z)$ satisfies

$$
\left|1+\frac{1}{2} \frac{z F^{\prime \prime}(z)}{F^{\prime}(z)}-\frac{z F^{\prime}(z)}{F(z)}\right|=\frac{1}{4}\left|\frac{z^{2}}{2+z}\right|<\frac{1}{4}
$$

$(z \in \mathbb{U})$.

For such a function $F(z)$, we see that

$$
\left|\frac{z^{2} F^{\prime}(z)}{F(z)^{2}}-1\right|=\left|e^{-z / 2}\left(1+\frac{1}{2} z\right)-1\right| \leqq c \quad(z \in \mathbb{U}) .
$$

By using the computer, we know that $c<0.18<1$. Indeed, the function $F(z)$ satisfying (97) implies that

$$
\operatorname{Re}\left(\frac{z F^{\prime}(z)}{F(z)}\right)>\frac{1}{2} \quad(z \in \mathbb{U}) .
$$

This shows us that $F(z) \in \mathcal{S}^{*}(1 / 2)$. 


\section{Competing Interests}

The authors declare that they have no competing interests.

\section{References}

[1] T. H. MacGregor, "A subordination for convex functions of order $\alpha$," Journal of the London Mathematical Society, vol. 9, no. 4, pp. 530-536, 1975.

[2] D. R. Wilken and J. Feng, "A remark on convex and starlike functions," Journal of the London Mathematical Society, vol. 21, no. 2, pp. 287-290, 1980.

[3] A. Marx, "Untersuchungen über schlichte Abbildungen," Mathematische Annalen, vol. 107, no. 1, pp. 40-67, 1933.

[4] E. Strohhäcker, "Beiträge zur Theorie der schlichten Funktionen," Mathematische Zeitschrift, vol. 37, no. 1, pp. 356-380, 1933.

[5] M. S. Robertson, "On the theory of univalent functions," Proceedings of the American Mathematical Society, vol. 37, no. 2, pp. 374-408, 1936.

[6] S. Owa, "On the distortion theorems," Kyungpook Mathematical Journal, vol. 18, no. 1, pp. 53-59, 1978.

[7] S. Owa, "On applications of the fractional calculus," Mathematica Japonica, vol. 25, no. 2, pp. 195-206, 1980.

[8] S. Owa and H. M. Srivastava, "Univalent and starlike generalized hypergeometric functions," Canadian Journal of Mathematics, vol. 39, no. 5, pp. 1057-1077, 1987.

[9] R. J. Libera, "Some classes of regular univalent functions," Proceedings of the American Mathematical Society, vol. 16, no. 4, pp. 755-758, 1965.

[10] P. L. Duren, Univalent Functions, Springer, New York, NY, USA, 1983.

[11] S. S. Miller and P. T. Mocanu, Differential Subordinations, vol. 225 of Pure and Applied Mathematics, Marcel Dekker, New York, NY, USA, 2000.

[12] S. S. Miller and P. T. Mocanu, "Second-order differential inequalities in the complex plane," Journal of Mathematical Analysis and Applications, vol. 65, no. 2, pp. 289-305, 1978.

[13] I. S. Jack, "Functions starlike and convex of order $\alpha$," Journal of the London Mathematical Society, vol. 2-3, no. 3, pp. 469-474, 1971.

[14] S. Ozaki and M. Nunokawa, "The Schwarzian derivative and univalent functions," Proceedings of the American Mathematical Society, vol. 33, pp. 392-394, 1972. 


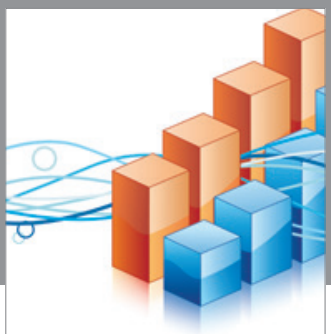

Advances in

Operations Research

vatem alat4

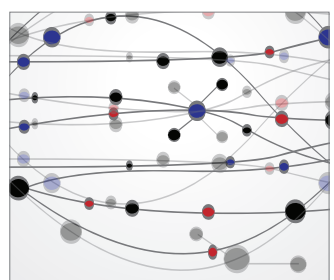

\section{The Scientific} World Journal
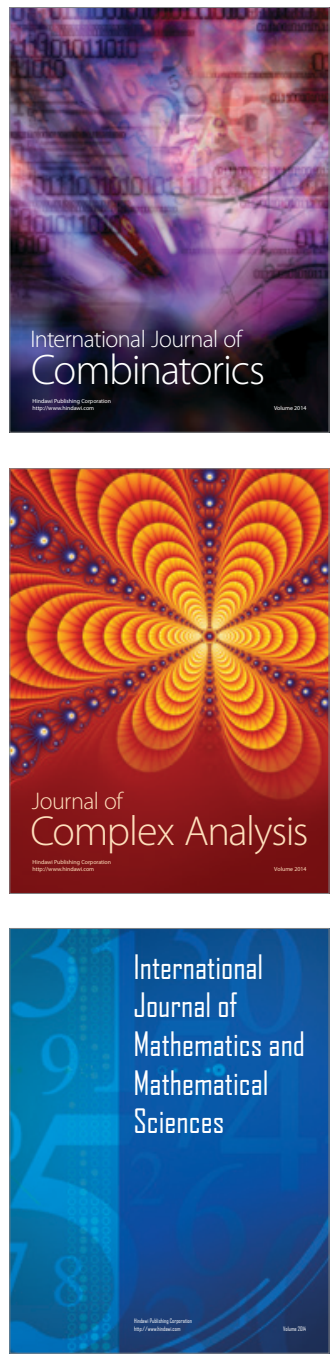
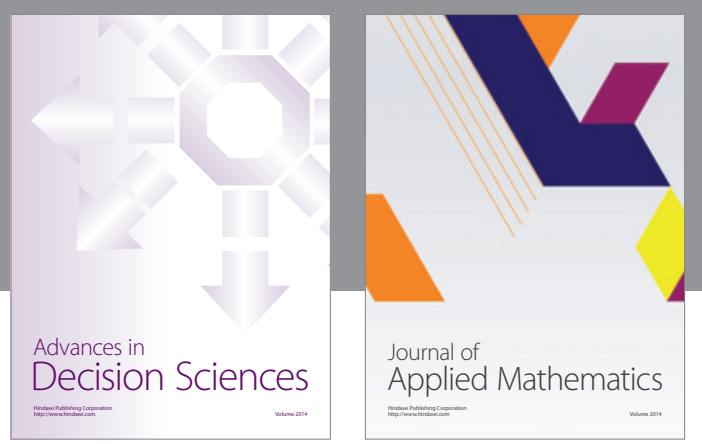

Algebra

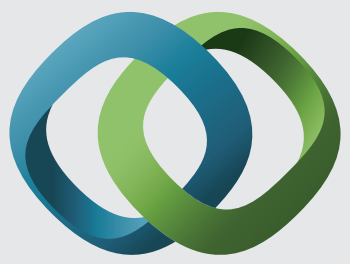

\section{Hindawi}

Submit your manuscripts at

http://www.hindawi.com
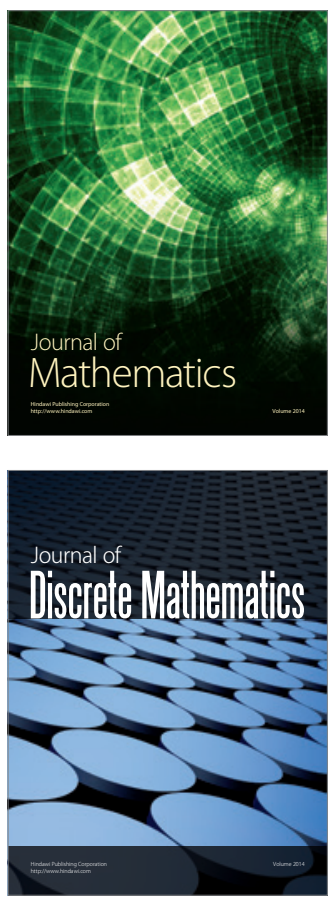

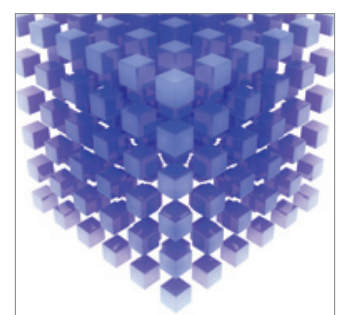

Mathematical Problems in Engineering
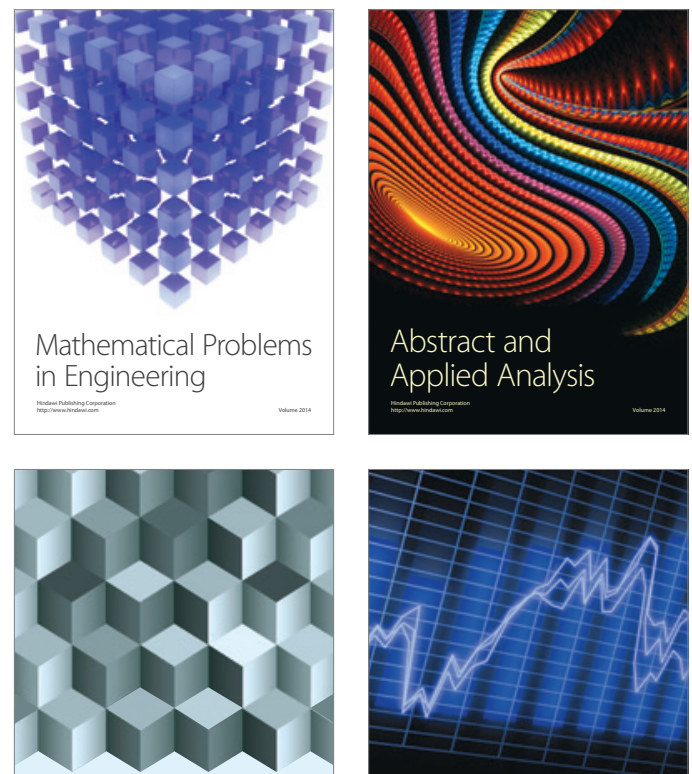

Journal of

Function Spaces

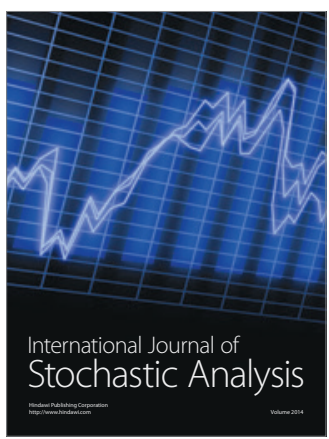

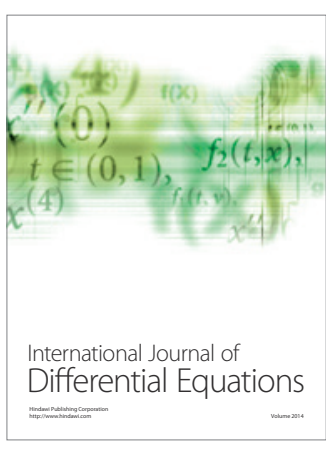
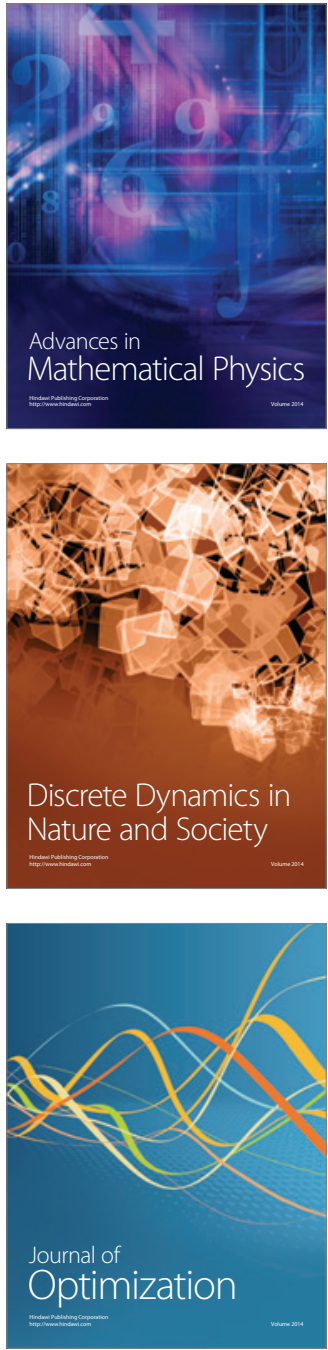